\title{
Mereological Destruction and Relativized Parthood: A Reply to Costa and Calosi
}

\author{
Jonathan D. Payton ${ }^{1}$ iD \\ Received: 27 May 2020 / Accepted: 17 May 2021 \\ (c) The Author(s), under exclusive licence to Springer Nature B.V. 2021
}

\begin{abstract}
Metaphysicians of various stripes claim that a single object can have more than one exact location in space or time - e.g. endurantists claim that an object persists by being 'all there' at different moments in time. Antony Eagle has developed a formal theory of location which is prima facie consistent with multi-location, but Damiano Costa and Claudio Calosi argue that the theory is unattractive to multi-location theorists on other grounds. I examine their charge that Eagle's theory won't allow an endurantist to account for certain cases of mereological change. I argue that the charge sticks, but not for the reasons Costa and Calosi think. Along the way, I explore an issue which is underexplored in their paper, namely, how an endurantist might modify Eagle's theory to incorporate a parthood relation which obtains, not absolutely, but only relative to times.
\end{abstract}

\section{Location and Multi-location}

Can a single object have more than one exact location in space or time? Many metaphysicians say 'Yes'. For instance, endurantists claim that an object persists through time by occupying successive moments in turn: if Tibbles the cat exists at $t_{1}$ and at $t_{2}$, then Tibbles herself is 'all there' at both $t_{1}$ and $t_{2}$.

But this idea is difficult to maintain. Indeed, some formal theories of location rule it out. For instance, Parsons (2007) develops a formal theory of location relations on which it's a theorem that every object has at most one exact location. ${ }^{1}$

Eagle (2010, 2016a, b) presents an alternative to such theories. Like Parsons, he begins with an ontology of regions (of space, time, or spacetime) which stand in relations analogous to familiar mereological ones - e.g. ' $s \leq r$ ' says that $s$ is a 'subregion' of $r$, where subregionhood, like parthood, is reflexive and transitive. He

1 Parsons (2007: 222-223; 228-229). Parsons also develops a theory similar to one found in Casati and Varzi (1999), in which the claim about exact location is an axiom.

Jonathan D. Payton

jonathanpayton@bilkent.edu.tr

1 Department of Philosophy, Bilkent University, Ankara, Turkey 
then takes a notion of 'occupation' as formally basic. Informally, $x$ occupies $r$-or, ' $\operatorname{Occ}(x, r)$ ' - just in case $x$ can be found, in whole or in part, at $r$. Finally, Eagle defines three further location relations in terms of occupation:

\section{Containment}

$$
\operatorname{Con}(x, r) \equiv_{\mathrm{df}} \forall y(y \leq x \supset \exists s(s \leq r \& \operatorname{Occ}(y, s)))
$$

\section{Filling}

$\operatorname{Fill}(x, r) \equiv_{\mathrm{df}} \forall s(s \leq r \supset \operatorname{Occ}(x, s))$

\section{Exact Location}

$$
\operatorname{Exact}(x, r) \equiv_{\mathrm{df}} \operatorname{Con}(x, r) \& \operatorname{Fill}(x, r) \& \sim \exists s(s<r \& \operatorname{Con}(x, s) \& \operatorname{Fill}(x, s))
$$

$x$ is contained at $r$ just in case every part of $x$ occupies a subregion of $r ; x$ fills $r$ just in case $x$ occupies every subregion of $r$, and $x$ is exactly located at $r$ just in case $x$ is contained in $r, x$ fills $\mathrm{r}$, and no proper subregion of $r$ both contains and is filled by $x$. On this theory, nothing prevents an object from having more than one exact location.

Unfortunately, Costa and Calosi (2020) say, Eagle's theory should still be unattractive to multi-location theorists of various stripes.

\section{Mereological Destruction}

Focusing on the endurantist, she presumably wants to allow than an object can endure through mereological change, i.e. that it can survive the gain and loss of parts. Now, consider a case of mereological destruction, in which an object loses a part between $t_{1}$ and $t_{2}$, and that part is completely destroyed. Poor Tibbles loses her tail between $t_{1}$ and $t_{2}$, and her tail is destroyed. Tibbles exists at both $t_{1}$ and $t_{2}$, but Tail exists only at $t_{1}$, not at $t_{2}$. Is Tibbles exactly located at both $t_{1}$ and $t_{2}$ ? The endurantist wants to say 'Yes'. But Eagle's theory says 'No'. Tibbles is exactly located at $t_{2}$ only if she's contained in $t_{2}$ - that is, only if all her parts occupy subregions of $t_{2}$. But while Tail is part of Tibbles, it doesn't exist at $t_{2}$, and so it occupies no subregion of $t_{2}$.

\section{Relativized Parthood: First Pass}

The obvious solution is to modify Eagle's theory by relativizing parthood (and other mereological relations) to regions of space, time, or spacetime. ${ }^{2}$ On this view, nothing is part of anything simpliciter, but only relative to some region. We could cash this out either by claiming that parthood is a three-place relation between two objects and a region, ${ }^{3}$ or by claiming that while parthood is a two-place relation between two objects, it's only instantiated relative to regions. I'll remain neutral on

\footnotetext{
${ }^{2}$ See, e.g., Simons (1987: ch.5) and Thomson (1983: 213-218).

${ }^{3}$ Or a four-place relation between two objects and two regions; see note 17.
} 
this issue, and simply use the labels ' $r$-parthood' and ' $\leq_{r}$ ' to denote this relativized parthood relation, however understood.

How does this modification help? If we replace parthood with $r$-parthood, we must revise Eagle's definition of containment:

\section{Containment 2}

$$
\operatorname{Con}(x, r) \equiv_{\mathrm{df}} \forall y\left(y \leq_{r} \supset \supset \exists s(s \leq r \& O c c(y, s))\right)
$$

$x$ is contained in $r$ just in case each of its $r$-parts occupies some subregion of $r{ }^{4}$ Now, suppose that Tail isn't a $t_{2}$-part of Tibbles (i.e., that it's a part of her relative to $t_{1}$, but not relative to $t_{2}$ ). Then, the endurantist can claim that Tibbles $i s$ exactly located at $t_{2}$, since Tibbles being contained in $t_{2}$ no longer requires that Tail occupies some subregion of $t_{2}$.

Why isn't Tail a $t_{2}$-part of Tibbles? Presumably, the answer has to do with how $r$-parthood is relativized to regions. When we set out to determine whether $y$ is an $r$-part of $x$, we're supposed to consider how, if at all, $x$ and $y$ are related to $r$. But is it the location of the whole which matters, or the location of the $r$-part (or both)? And what kind of location matters for $r$-parthood: occupation, containment, filling, or exact location?

If the relativization strategy is going to help with the problem of mereological destruction, then whether $y$ is an $r$-part of $x$ must depend at least on whether $y$ is suitably located at $r$. The endurantist's guiding thought seems to be that Tail isn't a $t_{2}$-part of Tibbles because (i) Tail doesn't exist at $t_{2}$ and (ii) being an $r$-part of $x$ at least requires existing at $r$ (Simons, 1987: 179; Thomson, 1983: 214-215). Since we already have relations between objects and times in our toolkit, it's natural to understand the notion of 'existence at a time' in terms of those relations: $y$ exists-at- $t$ just in case $y$ is suitably located at $t$. Generalizing to regions: $y$ exists-at $r$ just in case $y$ is suitably located at $r{ }^{5}$ So, whether $y$ is an $r$-part of $x$ must depend at least on whether and how $y$ is located at $r$.

For now, I'll assume that whether $y$ is an $r$-part of $x$ depends only on $y$ 's location relations to $r$, i.e. that $r$-parthood is relativized to the location of the part. If the endurantist modifies Eagle's theory using $r$-parthood, so understood, then she has four potential necessary conditions on $r$-parthood from which to choose ${ }^{6}$ :

\section{Occupation Condition}

$$
y \leq_{r} x \supset \operatorname{Occ}(y, r)
$$

\footnotetext{
${ }^{4}$ Here, I leave ' $\leq$ ' in place as a term for the two-place subregionhood relation. Even if we allow that mereological relations between objects are relative to regions, we needn't allow that the analogous relations between regions are relative in the same way.

5 Parsons (2007: 203) explicitly equates existing at a region with being located at that region. See also Donnelly (2011) and Gilmore (2006). Compare Costa (2017), Fine (2006), and Simons (2000: 422-425).

${ }^{6}$ She needn't choose only one; each principle is consistent with the others. Nor is she limited to these conditions; I'm only concerned with conditions which can help solve the problem of mereological destruction.
} 


\section{Containment Condition}

$y \leq_{r} x \supset \operatorname{Con}(y, r)$

Filling Condition

$y \leq_{r} \supset \supset \operatorname{Fill}(y, r)$

\section{Exact Location Condition}

$y \leq_{r} \supset \supset \operatorname{Exact}(y, r)$

Costa and Calosi suggest that the last of these is the most natural: when we evaluate whether $y$ is an $r$-part of $x$, "[t]he most natural candidate [for the relevant region] is the exact location of the object in question" (2020: 11). ${ }^{7}$ But, they object, this is circular. "Exact location is defined in terms of containment, and containment is defined in terms of parthood. Parthood, in turn, has to be relativized to exact locations. Hence, it seems that we need exact location in order to properly characterize containment, but we need containment in order to define exact location" (2020:11).

\section{Circularity Not the Issue}

The charge of circularity is too quick. Even if the endurantist adopts the Exact Location Condition, she needn't think that $r$-parthood must be characterized using the formal notion of exact location. She may take ' $\leq_{r}$ ' as basic and say that while the predicate 'Exact' figures in an axiom which governs the behaviour of ' $\leq_{r}$, , it doesn't figure in any definition or analysis of ' $\leq_{r}$.

There might still be a sense in which we rely on the notion of exact location to grasp the notion of $r$-parthood. Although ' $\leq_{r}$ ' is formally basic, we might need a notion of exact location in order to grasp the informal notion of $r$-parthood. (E.g. we might rely on our sense of whether $y$ is exactly located at $r$ in order to judge whether $y$ is part of $x$, relative to $r$.) But the notion of exact location on which we rely might itself be an informal notion, rather than the formal one given by ' $y \leq_{r} x{ }^{\text {' }}{ }^{8}$ That we rely on such an informal notion of exact location to understand the informal notion of $r$-parthood is no objection to taking ' $\leq_{r}$ ' as basic. (Compare: many find the reflexive notion of parthood, ' $\leq$ ', counter-intuitive at first. Their intuitive notion of a part corresponds to the formal notion of a proper part, ' $<$ ', and they understand the reflexive notion informally as '...is either part of or identical to...' This is no objection to taking ' $\leq$ ' as basic and defining ' $<$ ' in terms of it.)

\footnotetext{
7 They might mean to suggest that it's the exact location of the whole which matters: $y$ is an $r$-part of $x$ only if $x$ is exactly located at $r$. But while the endurantist may accept this as an additional condition on $r$-parthood - see Sect. 7 - it won't help her solve the problem of mereological destruction on its own, since she thinks that Tibbles is exactly located at $t_{2}$.

${ }^{8}$ E.g., we may rely on Gilmore's (2006: 200) characterization: $x$ is exactly located at $r$ just in case $x$ has (or has-at- $r$ ) the exact shape and size as $r$, and for any $y, x$ stands (or stands-at- $r$ ) in all the same spatial or spatiotemporal relations to $y$ as $r$ does.
} 


\section{Triviality}

While the charge of circularity is too quick, there are other problems with the view.

First, the right-hand side of Containment 2 is trivially true if $x$ has no $r$-parts. If $x$ has no $r$-parts, then trivially, all of them occupy some subregion of $r$. Thus, $x$ is contained in all the regions where it has no parts.

Worse still, if we combine Containment 2 with any of the four conditions on $r$-parthood, the right-hand side will be true for any arbitrary $x$ and $r$.

\section{Trivial Containment}

$\operatorname{Con}(x, r)$

That is, every object will be contained in every region.

Take the Occupation Condition first. According to it, $y$ is an $r$-part of $x$ only if $y$ occupies $r$. Since subregionhood is reflexive, it follows that $y$ is an $r$-part of $x$ only if $y$ occupies some subregion of $r$ (namely, $r$ ).

(1) $y \leq_{r} x \supset \exists s(s \leq r \& O c c(y, s))$.

(1) is just the right-hand side of Containment 2. So, for any $x$ and $r$, we can infer that $x$ is contained in $r$. Hence, Trivial Containment.

A similar proof works for the Filling Condition. ${ }^{9}$ And, since exact location implies filling, combining Containment 2 with the Exact Location Condition leads to Trivial Containment, too.

That leaves the Containment Condition. Trivial Containment doesn't follow from this condition, given only what we have so far. But it will follow if we add another plausible principle.

If $r$-parthood is really a parthood relation, it should obey a reflexivity axiom. Granting that objects have parts only relative to regions, we can't say that any $x$ is part of itself relative to any arbitrary $r$, since $x$ needn't have any parts relative to $r$. (Tail doesn't have any parts relative to $t_{2}$, even itself. ${ }^{10}$ Fortunately for the endurantist, there's a plausible alternative:

\section{Reflexivity}

$\exists x\left(y \leq_{r} x\right) \supset y \leq_{r} y$

If $y$ is part of anything relative to $r$, then it's part of itself relative to $r$.

\footnotetext{
9 According to the Filling Condition, $y$ is an $r$-part of $x$ only if $y$ occupies every subregion of $r$. Since subregionhood is reflexive, (1) follows as above.

10 Compare Thomson (1983: 216). This raises another issue for the endurantist. Given Containment, containment implies occupation, as it should, by the reflexivity of (unrelativized) parthood. If we switch to Containment 2, we don't get this result, since $r$-parthood isn't reflexive.
} 
But now, suppose the endurantist adopts the Containment Condition. By Containment 2, $y$ is an $r$-part of $x$ only if each of $y$ 's $r$-parts occupies some subregion of $r$ :

(2) $y \leq_{r} x \supset \forall z\left(z \leq_{r} y \supset \exists s(s \leq r \& \operatorname{Occ}(z, s))\right)$.

Given Reflexivity, if $y$ is an $r$-part of $x$ then $y$ is an $r$-part of itself. But then, by (2), if $y$ is an $r$-part of $x$ then $y$ occupies some subregion of $r$. That's just (1), which implies Trivial Containment. ${ }^{11}$

\section{Weakness}

The endurantist can avoid both problems from the previous section by adding to Containment 2 an extra condition to the effect that $x$ has at least one $r$-part:

\section{Containment 3}

$$
\operatorname{Con}(x, r) \equiv_{\mathrm{df}} \exists y\left(y \leq_{r} x\right) \& \forall z\left(z \leq_{r} x \supset \exists s(s \leq r \& \operatorname{Occ}(z, s))\right)
$$

Now, if $x$ has no $r$-parts then the conclusion that $x$ is contained in $r$ is blocked rather than trivialized. And the arguments for Trivial Containment are all blocked.

A problem remains, however. The second conjunct of Containment 3 -i.e. Containment 2 - still holds for any $x$ and $r$, given any of our four conditions on $r$-parthood. ${ }^{12}$ So, what Containment 3 amounts to is that $x$ is contained in $r$ just in case it has at least one $r$-part ${ }^{13}$ :

$$
\begin{aligned}
& \text { Weak Containment } \\
& \operatorname{Con}(x, r) \equiv \exists y\left(y \leq_{r} x\right)
\end{aligned}
$$

\footnotetext{
11 Thomson (1983: 216) claims that $x$ is part of itself relative to any time at which it exists. Generalizing to regions: $x$ is part of itself relative to any region at which it exists. We can give a variant of the argument using this reflexivity axiom. If parthood is relativized to regions, then being an $r$-part of $x$ requires existing at $r$. Thus, if $y$ is an $r$-part of $x$, then $y$ exists at $r$, and so (by Thomson's reflexivity axiom) $y$ is an $r$-part of itself. By (2), it follows that if $y$ is an $r$-part of $x$ then $y$ occupies some subregion of $r$.

12 The arguments from the Occupation, Filling, and Exact Location conditions proceed as in the previous section. The argument from the Containment Condition relied on Containment 2, but a variant uses Containment 3.

According to the Containment Condition, any $r$-part of $x$ is contained in $r$. By Containment 3, if $y$ is an $r$-part of $x$, then $y$ itself has at least one $r$-part, and each of its $r$-parts occupies a subregion of $r$ :
}

$$
y \leq_{r} x \supset\left(\exists z\left(z \leq_{r} y\right) \& \forall v\left(v \leq_{r} y \supset \exists s(s \leq r \& \operatorname{Occ}(v, s))\right)\right) .
$$

By Reflexivity, if $y$ is an $r$-part of $x$, then $y$ is an $r$-part of itself. So, if $y$ is an $r$-part of $x$, then $y$ occupies some subregion of $r$. That's just (1), which is the second conjunct of Containment 3.

13 Eagle (2019: 176) notes that something like Weak Containment follows even from Containment 2. My discussion shows that the problems for Containment $\mathbf{2}$ are even worse than this, and that the natural way of avoiding those problems still gives rise to Weak Containment. 
This is a bad result, given the assumption from Sect. 3 that $r$-parthood is relativized to the location of the part. Given that assumption, the fact that $x$ has an $r$-part shouldn't imply that $x$ itself is contained in that region: if I stick my arm into the hallway outside my office, then my arm is a part of me relative to (some subregion of) the hallway; it shouldn't follow that I'm contained in (that subregion of) the hallway, since I have parts that don't occupy the hallway.

\section{Relativized Parthood: Second Pass}

Perhaps it's the assumption from Sect. 3 that's causing the trouble. While $r$-parthood must be relativized at least to the location of the part, if it's to be of any use to the endurantist in solving the problem of mereological destruction, perhaps we should relativize $r$-parthood to the locations of the part and the whole.

There's some independent motivation for this approach to $r$-parthood. Compare the view that parthood is relativized to regions with the view that property instantiation more generally is so relativized. On this view, no object possesses any property simpliciter, but only relative to some region of space, time, or spacetime. ${ }^{14}$ Assume that view is right. To which region should the instantiation of a property by an object be relativized?

In some cases, the natural answer will be, not the (or an) exact location of the object, but a proper subregion of that location. Consider a checkerboard made up of red and black squares. It seems that the checkerboard instantiates redness only with respect to certain subregions of its exact location, namely the exact locations of the red squares plus some (and perhaps all) subregions of those locations. After all, those are the regions with respect to which we're inclined to say, 'The checkerboard is red here'.

In other cases, the natural answer to the question 'With respect to which region does $x$ instantiate $F$ ?' is $x$ 's exact location. Consider again the checkerboard, and let $r$ be some region with respect to which it instantiates redness. It seems that the checkerboard instantiates the property being red at $r$ at its exact location, not at any proper subregion of it. That property is one the checkerboard 'simply has', or 'has as a whole', as opposed to one which it has at some parts of its surface and not others. The instantiation-relativization theorist cashes this out is by saying that the checkerboard instantiates that property at its exact location, rather than at any proper subregion of it.

Now consider relations. If $x$ bears $R$ to $y$, we can ask, 'With respect to which region does $x$ bear $R$ to $y$ ?' In many cases, the natural answer seems to be the sum of $x$ 's and $y$ 's exact locations. Suppose that $x$ is taller than $y$, and let $r_{x}$ and $r_{y}$ be their exact locations. It certainly seems arbitrary to relativize the instantiation of taller than to just one of $r_{x}$ and $r_{y}$. (Which one?) And there's little reason to relativize its instantiation to the sum of some proper subregions of $r_{x}$ and $r_{y}$. Rather, taller than

14 See, e.g., Johnston (1987: 125-129) and van Inwagen (1990: 249-250). 
seems to be a relation that $x$ and $y$ 'simply have', so the natural thing to say is that they instantiate it relative to the sum of $r_{x}$ and $r_{y}{ }^{15}$

This seems to be the case for parthood. If $y$ is part of $x$, then parthood seems to be a relation that $y$ and $x$ 'simply have', so its instantiation should be relativized to the sum of their exact locations.

There's one more complication to add to this picture. Intuitively, if $y$ is part of $x$, then its exact location is a subregion of $x$ 's exact location (Casati \& Varzi, 1999: ch.2; Markosian, 2014: 72-74; McDaniel, 2014: 20). So, we have:

\section{Sum Condition}

$$
y \leq_{r} x \supset \exists s_{1} \exists s_{2}\left(s_{1} \leq s_{2} \& r=s_{1}+s_{2} \& \operatorname{Exact}\left(y, s_{1}\right) \& \operatorname{Exact}\left(x, s_{2}\right)\right)
$$

$y$ is an $r$-part of $x$ only if $y$ and $x$ are exactly located at some regions $s_{1}$ and $s_{2}$, respectively, such that $s_{1}$ is a subregion of $s_{2}$ and $r$ is the sum of $s_{1}$ and $s_{2}$. Of course, if $s_{1}$ is a subregion of $s_{2}$, then the sum of $s_{1}$ and $s_{2}$ is just $s_{2}$. So, Sum Condition is equivalent to:

\section{Sum Condition 2}

$$
y \leq_{r} x \supset(\operatorname{Exact}(x, r) \& \exists s(s \leq r \& \operatorname{Exact}(y, s)))
$$

$y$ is an $r$-part of $x$ only if $x$ is exactly located at $r$ and $y$ is exactly located at some subregion of $r{ }^{16,17}$

Sum Condition 2 has a lot going for it. Moreover, while it still implies Weak Containment when combined with Containment $3,{ }^{18}$ that result is no longer problematic. Recall the case where I stick my arm into the hallway. Assuming that parthood is relativized just to a location of the part, and hence that my arm is a part of me relative to (some subregion of) the hallway, we get the bad result that I'm contained in (that subregion of) the hallway. But given Sum Condition 2, my arm is not a part of me relative to (some subregion of) the hallway. Rather, it's a part of me relative to the sum of my exact location and my arm's exact location. And we should expect me to be contained in that region. Generalizing: given Sum Condition 2, we should expect an object to be contained wherever it has $r$-parts.

\footnotetext{
15 McDaniel (2014: 20) makes a similar argument.

16 McDaniel (2014: 20) makes this point, but doesn't use it (as I'm about to) to raise trouble for any theory of relativized-parthood + multiple-location.

17 Gilmore (2009: 101-109) considers a view like this and rejects it on the ground that there's no 'simple and straightforward' transitivity principle for $r$-parthood so understood. (The simplest principle, 'If $x$ is an $r$-part of $y$, and $y$ is an $s$-part of $z$, then $x$ is an $s$-part of $z$, fails if objects can be multiply located in time and survive mereological destruction. Tail is a $t_{1}$-part of Tibbles' back half, and Tibbles' back half is a $t_{2}$-part of Tibbles, but Tail is not a $t_{2}$-part of Tibbles.) Gilmore (2009: 113-131) develops an alternative on which parthood is four-place, relativized to both the exact location of the part and the exact location of the whole, rather than to their sum. Kleinschmidt (2011: 257-261) argues that this alternative still has problems with transitivity.

18 Sum Condition 2 implies that $y$ is an $r$-part of $x$ only if $y$ is exactly located at a subregion of $r$. It follows that $y$ is an $r$-part of $x$ only if $y$ occupies a subregion of $r$. That's just (1), which is the second conjunct of Containment 3. Hence, Weak Containment.
} 


\section{No Mere Containment}

But a problem remains. By Containment 3, $x$ is contained in $r$ only if $x$ has at least one $r$-part. But according to Sum Condition 2, if $x$ has at least one $r$-part then $x$ is exactly located at $r$. So, $x$ is contained in $r$ only if $x$ is exactly located at $r$.

\section{No Mere Containment}

$\operatorname{Con}(x, r) \supset \operatorname{Exact}(x, r)$

This is a bad result: I'm currently contained my office, but not exactly located there, since I don't occupy every subregion of it.

\section{Summing Up}

Costa and Calosi are right that Eagle's theory, as he originally states it, won't get an endurantist everything she presumably wants. In particular, it won't allow her to say that objects can endure through mereological destruction. They're also right that the endurantist can't solve this problem in the obvious way, by relativizing parthood to regions. But the reason the relativization strategy fails isn't that it lands the endurantist in circularity, but rather that (in the absence of an alternative approach to those discussed here) it commits her to false claims in the theory of location.

Acknowledgments This discussion piece began as a commentary on Damiano Costa and Claudio Calosi's "The multi-location trilemma", given at the 2019 meeting of the Society for the Metaphysics of Science in Toronto, Canada. Thanks to the audience at that meeting, to Damiano and Claudio for discussion, and to Tuomas E. Tahko for the invitation to give comments. Thanks also to two anonymous referees for this journal for their very helpful feedback. Research was partially funded by a Postdoctoral Fellowship from the Social Sciences and Humanities Research Council of Canada.

\section{References}

Casati, R., \& Varzi, A. (1999). Parts and places: The structures of spatial representation. The MIT Press. Costa, D. (2017). The transcendentist theory of persistence. Journal of Philosophy, 114(2), 57-75.

Costa, D., \& Calosi, C. (2020). The multi-location trilemma. Erkenntnis. https://doi.org/10.1007/ s10670-020-00230-7

Donnelly, M. (2011). Endurantist and perdurantist accounts of persistence. Philosophical Studies, 154(1), 27-51.

Eagle, A. (2010). Location and perdurance. In D. Zimmerman (Ed.), Oxford studies in metaphysics (Vol. 5, pp. 53-94). Oxford University Press.

Eagle, A. (2016a). Persistence, vagueness, and location. Journal of Philosophy, 113(10), 507-532.

Eagle, A. (2016b). Multiple location defended. Philosophical Studies, 173(8), 2215-2231.

Eagle, A. (2019). Weak location. Dialectica, 73(1-2), 149-181.

Fine, K. (2006). In defense of three-dimensionalism. Journal of Philosophy, 103(12), 699-714.

Gilmore, C. (2006). Where in the relativistic world are we? Philosophical Perspectives, 20, 199-236.

Gilmore, C. (2009). Why parthood might be a four-place relation, and how it behaves if it is. In L. Honnefelder, B. Schick, \& E. Runggaldier (Eds.), Unity and time in metaphysics (pp. 83-133). de Gruyter.

Johnston, M. (1987). Is there a problem about persistence? Proceedings of the Aristotelian Society, Supplementary, 61, 107-135. 
Kleinschmidt, S. (2011). Multilocation and mereology. Philosophical Perspectives, 25(1), 253-276.

Markosian, N. (2014). A spatial approach to mereology. In S. Kleinschmidt (Ed.), Mereology and location (pp. 69-90). Oxford University Press.

McDaniel, K. (2014). Parthood is identity. In S. Kleinschmidt (Ed.), Mereology and location (pp. 13-32). Oxford University Press.

Parsons, J. (2007). Theories of location. In D. Zimmerman (Ed.), Oxford studies in metaphysics (Vol. 3, pp. 201-232). Oxford University Press.

Simons, P. (1987). Parts: A Study in Ontology. Oxford University Press.

Simons, P. (2000). How to exist at a time when you have no temporal parts. The Monist, 83(3), 419-426. Thomson, J. J. (1983). Parthood and identity across time. Journal of Philosophy, 80(4), 201-220. van Inwagen, P. (1990). Four-dimensional objects. Noûs, 24(2), 245-255.

Publisher's Note Springer Nature remains neutral with regard to jurisdictional claims in published maps and institutional affiliations. 